\title{
WAVE PROPAGATION IN EXCITABLE MEDIA WITH RANDOMLY DISTRIBUTED OBSTACLES*
}

\author{
K. H. W. J. TEN TUSSCHER ${ }^{\dagger}$ AND A. V. PANFILOV $\ddagger$
}

\begin{abstract}
We study the effect of small, randomly distributed obstacles on wave propagation in two-dimensional (2D) and 3D excitable media described by the Aliev-Panfilov model. We find that increasing the number of obstacles decreases the conduction velocity of plane waves and decreases the effective diffusion coefficient in the eikonal curvature equation. The presence of obstacles also increases the inducibility of wave breaks and spiral waves in 2D and 3D excitable media, but suppresses spiral breakup induced by a steep restitution curve mechanism. We discuss the mechanisms of the observed effects, the differences between $2 \mathrm{D}$ and 3D excitable media, as well as the relevance of our study to processes of wave propagation in cardiac tissue, including arrhythmogenesis in the presence of fibrosis in the myocardium.
\end{abstract}

Key words. excitable media, spiral waves, obstacles, wave break, discontinuous conduction, cardiac arrhythmias, fibrosis

AMS subject classification. $74 \mathrm{~J} 30$

DOI. $10.1137 / 030602654$

1. Introduction. One of the most fundamental processes which occur in reaction-diffusion excitable systems is propagation of nonlinear waves. Examples of such waves include chemical waves in the Belousov-Zhabotinsky reaction [46]; waves of $\mathrm{CO}$ oxidation on Pt catalytic surfaces [19]; electrical waves in retinal and cortical nerve tissue [15], where they may underlay neurological diseases such as epilepsia; and waves in heart muscle, where they may be involved in life-threatening cardiac arrhythmias $[3,10]$. In addition, nonlinear waves control the morphogenesis of Dictyostelium discoideum amobae [14] and initiate the development of Xenopus oocytes after fertilization [28]. Nonlinear waves in excitable media have been studied in numerous analytical and numerical studies (see [16, 48, 32] for an overview). Most properties of nonlinear waves in excitable media, such as propagation with a constant speed that is independent from initial conditions, the annihilation of colliding waves, the dependence of propagation speed on wave front curvature, as well as the possibility of formation of rotating spiral waves (vortices) are general for all excitable systems listed above and follow from a general mathematical description of excitable systems [27].

One of the important questions concerning nonlinear waves is their propagation in the presence of obstacles. One particular aspect of this question, the influence of a single or few large obstacles on wave propagation in excitable media, has been extensively studied over the years. It was shown that obstacles affect various aspects of wave propagation, including wave front shape, and that they can induce spiral waves through vortex shedding or by causing localized conduction block in narrow isthmuses

*Received by the editors December 22, 2003; accepted for publication (in revised form) June 7, 2004; published electronically February 9, 2005. This research is funded by the Netherlands Organization for Scientific Research (NWO) through grant 620061351 of the Research Council for Physical Sciences (EW).

http://www.siam.org/journals/mms/3-2/60265.html

${ }^{\dagger}$ Department of Theoretical Biology, Utrecht University, Padualaan 8, Utrecht, $3584 \mathrm{CH}$, The Netherlands (khwjtuss@hotmail.com).

${ }^{\ddagger}$ Department of Theoretical Biology, Utrecht University, Padualaan 8, Utrecht, $3584 \mathrm{CH}$, The Netherlands, and Division of Mathematics, University of Dundee, 23 Perth Road, Dundee DD1 4HN, United Kingdom (panfilov@maths.dundee.ac.uk). 
formed between obstacles [36, 1, 7, 8, 12]. In addition, obstacles can stabilize spiral wave rotation by anchoring the spiral wave [10, 39].

Another important question is what happens to wave propagation in the presence of large numbers of small obstacles, when the collective effects of multiple obstacles rather than the interaction of waves with solitary obstacles are of importance. This type of situation is particularly important in cardiac excitable tissue. In recent years it has become clear that there is a strong correlation between the increased presence of inexcitable fibrotic tissue in the heart and the occurrence of dangerous cardiac arrhythmias. Fibrotic tissue consists of specialized cells, called fibroblasts, and the materials these cells excrete (collagen, elastin). These materials make up the socalled extracellular matrix, which serves to hold the cells of the heart in place [22]. Fibrotic tissue thus plays an important role in determining the structure of cardiac tissue, but since fibroblasts and extracellular matrix are nonexcitable and insulated, they do not conduct electrical waves, and thus form obstacles for waves propagating through the heart. In healthy hearts fibrotic tissue makes up only $5 \%$ of the total tissue, but during the process of aging, in particular cardiac diseases related to heart failure (cardiac myopathies), and after an infarction, this may increase up to $35 \%$. All these conditions that lead to an increase of fibrotic tissue are also linked to a much larger incidence of cardiac arrhythmias, thus suggesting a potential causal relationship $[11,45,42,18,44,17,22]$. Whereas after infarction, fibrotic tissue increases locally and appears in the form of long fibrous strands, during aging or in certain diseases, fibrotic tissue is present in a more diffuse form, forming small randomly dispersed patches.

From a mathematical point of view dispersed fibrotic tissue serves as multiple obstacles for wave propagation in cardiac tissue. Fibrotic strands can be modeled as long one-dimensional (1D) obstacles, whereas diffuse fibrosis can be represented as multiple small obstacles randomly distributed over the cardiac tissue. The basic ideas for modeling the effects of multiple objects were developed in [38], which introduced a model of textured cardiac tissue as an excitable medium containing multiple inexcitable obstacles. It was demonstrated that waves propagating in such media can induce an electrogram characteristic for discontinuous propagation occurring in fibrotic cardiac tissue. However, this paper dealt with the introduction of a new model rather than its detailed study and was limited to several important but illustrative examples.

In this article we perform a detailed study on the influence of randomly distributed small obstacles on several important aspects of wave propagation. We study the effect of obstacles on planar wave propagation speed and on the eikonal curvature relation in $2 \mathrm{D}$ media. We investigate the influence of obstacles on the vulnerability for spiral wave generation in 2D and 3D media. Finally, we study the effect of obstacles on the onset of spatio-temporal chaos through spiral wave breakup in $2 \mathrm{D}$ and $3 \mathrm{D}$ media. A part of this research was published as a brief report in [43].

2. Model. The excitable medium is described by the Aliev-Panfilov model [2]:

$$
\begin{aligned}
& \frac{\partial e}{\partial t}=-k e(e-a)(e-1)-e r+\Delta_{2,3} e, \\
& \frac{\partial r}{\partial t}=\left(\epsilon+\frac{\left(\mu_{1} r\right)}{\left(\mu_{2}+e\right)}\right)(-r-k e(e-b-1)) .
\end{aligned}
$$

Here the variable $e$ stands for the transmembrane potential; $\Delta$ is a $2 \mathrm{D}\left(\Delta_{2}=\right.$ $\left.\partial^{2} / \partial x^{2}+\partial^{2} / \partial y^{2}\right)$ or $3 \mathrm{D}\left(\Delta_{3}=\partial^{2} / \partial x^{2}+\partial^{2} / \partial y^{2}+\partial^{2} / \partial z^{2}\right)$ Laplace operator; and 
the variable $r$ stands for the conductance of the slow inward current. The expression $-k e(e-a)(e-1)$ in $(2.1)$ determines the fast processes, such as the upstroke of the action potential. The dynamics of the recovery phase of the action potential are determined by the time course of the variable $r$, mainly by the expression $\left(\epsilon+\left(\mu_{1} r\right) /\left(\mu_{2}+e\right)\right)$. The parameter values used in this study are $a=0.1, \mu_{2}=0.3$, $k=8, \epsilon=0.01, b=0.1$, while parameter $\mu_{1}$ is varied.

In two dimensions Laplacians were numerically approximated using 4-point centered difference Euler schemes; in three dimensions 6-point centered difference schemes were used. Computations were performed using an explicit time integration scheme with a time integration step of $\Delta t=0.2$ and space integration step of $\Delta x=0.6$ (both dimensionless units), as in previous publications [34]. No-flux boundary conditions were imposed. In two dimensions a $200 \times 200$ sheet was used; in three dimensions $100 \times 100 \times 40,100 \times 100 \times 60$ or $200 \times 200 \times 76$ slabs were used. Nonexcitable cells were modeled as $1 \times 1$ obstacles with Neumann boundary conditions, similar to [38, 1], and were randomly distributed over the medium. To quantitatively investigate the effect of the obstacles, we varied the percentage of grid points in the medium occupied by obstacles, and refer to this as the percentage obstacles. The size of the obstacles, scaled on the basis of a spiral wave length of $30 \mathrm{~mm}$ in human cardiac tissue, was $1 \times 1 \mathrm{~mm}^{2}$. To initiate the first spiral wave we used initial data corresponding to a broken wave front, the break being located at the middle of the excitable medium. If a spiral persisted for 80 cycles without fragmentation, we considered breakup to be absent for these parameter values.

\section{Wave propagation in $2 \mathrm{D}$ media.}
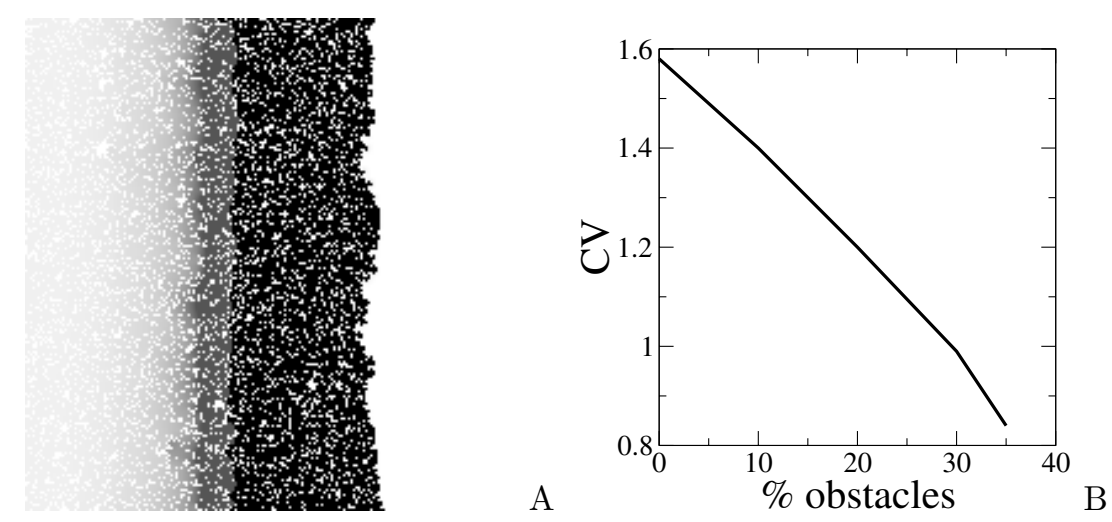

FIG. 3.1. (A) Planar wave front propagating from left to right in a medium with $20 \%$ obstacles. The black area represents the excited state $(e>0.6)$, and intermediate shading from gray to white shows different levels of recovery. (B) Dependence of planar wave conduction velocity $(C V)$ on the percentage of obstacles. Results are for $\mu_{1}=0.13$.

3.1. Plane wave propagation in two dimensions. First we study the effect of randomly distributed nonexcitable obstacles on planar wave conduction velocity (CV) in 2D excitable media. For that we stimulated an excitable medium along the left vertical boundary and measured the velocity of the initiated wave. In an excitable medium without obstacles the wave front will be along a straight vertical line, and conduction velocity can be evaluated from simple 1D computations. However, the presence of obstacles will disturb the wave front shape (Figure 3.1(A)), and therefore conduction velocity should be measured as an average along the vertical axis. Figure 

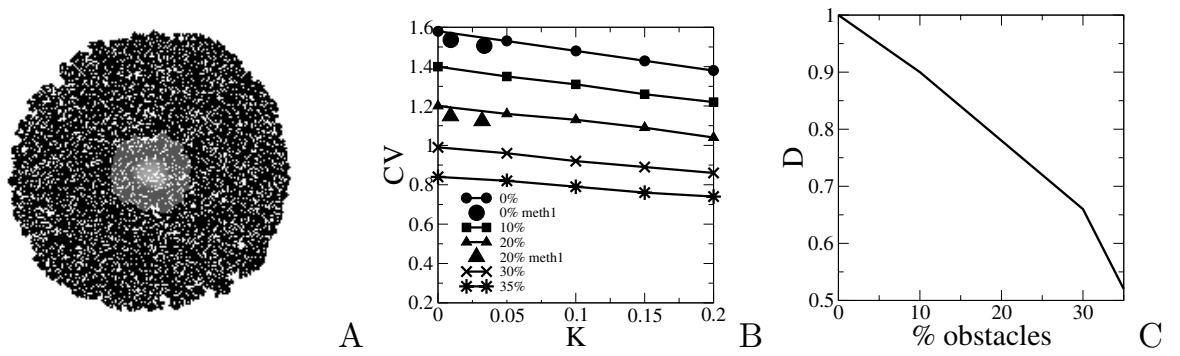

FIG. 3.2. (A) A circular wave front in a medium with $30 \%$ obstacles. (B) Dependence of conduction velocity $(C V)$ on wave front curvature $(K)$ for different percentages of obstacles present. Most data are obtained using the second method described; for $0 \%$ and $20 \%$ of obstacles some data are also obtained using the first method and are added for comparison. (C) Dependence of the diffusion coefficient on the percentage of obstacles. Results are for $\mu_{1}=0.13$. For further explanation, see text.

3.1(B) shows the dependence of average planar CV on the percentage of obstacles. It can be seen that $\mathrm{CV}$ decreases as a function of the percentage of nonexcitable obstacles present in the medium. We also see that this dependence can be well approximated by a straight line. When the percentage of obstacles exceeded $38 \%$, we observed failure of wave propagation.

3.2. Curvature effects on wave speed in two dimensions. As a next step we investigate how the presence of randomly distributed small obstacles influences the eikonal curvature relation in 2D excitable media. As was shown in [47], the speedcurvature relation in a $2 \mathrm{D}$ excitable medium is given by the following formula, valid for small curvatures:

$$
C V=C V_{0}-D K
$$

where $C V_{0}$ is the conduction velocity of a planar wave, $D$ is the diffusion coefficient of the medium, and $K$ is the wave front curvature. We studied the relation between wave speed and wave front curvature for different percentages of obstacles.

There are two possible ways of computing the eikonal curvature relation. One method is the direct measurement of propagation velocity in a $2 \mathrm{D}$ computation of an expanding radial wave. Because the velocity of the wave changes continuously with the wave front curvature (which changes because of the expansion of the radial front), this method requires high accuracy. However, a single simulation results in the complete curvature relation. The other method is to determine the curvature relation from waves propagating with constant curvature and velocity, which mathematically can be studied as waves propagating in a medium with a modified diffusion term: $\frac{\partial^{2}}{\partial x^{2}}$ in (2.1) is replaced by $\frac{\partial^{2}}{\partial x^{2}}+K \frac{\partial}{\partial x}$, where $K$ is the constant wave front curvature [24, 31]. Because wave front curvature and speed stay constant during a single simulation, this method requires much less accuracy. The complete curvature relation can be obtained by varying $K$ and determining constant wave speed.

In Figure 3.2(A) we can see a snapshot of an expanding radial wave in an excitable medium with obstacles, initiated by applying a point stimulus in the center of the medium. We see that the wave has an approximately circular-shaped wave front; however, the presence of obstacles causes substantial local disturbances of the wave front shape. These local disturbances will cause inevitable errors in determining wave speed, which should be determined instantly for each position of the wave front when 
using the first method for determining the eikonal curvature relation. We therefore decided to use the second method.

In Figure 3.2(B) we show the dependence of the CV on curvature for different percentages of obstacles found using this second method. For comparison purposes, for 0 and $20 \%$ obstacles a few data points obtained using the first method are also shown. Two observations can be made from this figure. First, an increase in the percentage of obstacles leads to a decrease of $\mathrm{CV}$, both in the absence and presence of curvature. Second, an increase in the wave front curvature leads to a linear decrease in wave front propagation speed, both in the absence and presence of obstacles; thus the linear dependence in the eikonal curvature equation (3.1) also holds in the presence of obstacles. In addition, it can be seen that the data points obtained using the first method have values similar to those obtained using the first method. Finally, we determined the effective diffusion coefficient, which determines the slope of the $C V$ versus $K$ curve. In Figure 3.2(C) we plot this diffusion coefficient as a function of the percentage of obstacles. It can be seen that the diffusion coefficient decreases almost linearly with the percentage of obstacles. This decrease can be explained by the effective decrease of coupling between excitable cells in the presence of obstacles.

Note that the linear decrease of the effective diffusion coefficient alone (Figure $3.2(\mathrm{C})$ ) cannot explain the linear decrease in wave velocity in (Figure 3.1(B)), since the velocity of a wave in an excitable medium is proportional to the square root of the diffusion coefficient. This indicates that the obstacles also change the effective reaction rate of the medium. These effects can possibly be studied analytically using homogenization theory of excitable media [25], as was shown in [6] for the case of small periodic obstacles that are nonreactive, but allowing for diffusion.

3.3. Vulnerability in the presence of obstacles in two dimensions. When extra stimuli are applied in the heart in the interval between heart beats, there is a window of time during which life-threatening arrhythmias can be induced. This window is called the vulnerable period or the vulnerable window. The vulnerable period can be readily demonstrated in any model of an excitable medium that is heterogeneous with respect to the refractory period [26]. In such models the extra stimulation can induce wave breaks that evolve into rotating spiral waves of excitation, which are believed to underlie particular types of cardiac arrhythmias called tachycardias. In addition, it has been shown that high frequency stimulation can also lead to spiral wave formation in the presence of inexcitable obstacles [36, 1, 7, 8, 38].

Here we study the vulnerability of $2 \mathrm{D}$ excitable media that are homogeneous in their properties of excitability and refractoriness but contain randomly distributed obstacles. To do so we apply three subsequent stimuli with a given coupling interval along the left vertical boundary of the medium, and study whether they will induce wave breaks and spiral waves. In a homogeneous excitable medium without obstacles this method of stimulation will lead either to normal wave propagation, or, if the period of stimulation is too low and cells have not recovered yet, to the propagation block of one or several waves. However, in the presence of obstacles, there is a third possibility, namely, that wave propagation blocks locally and progresses in other places, thus leading to the formation of wave breaks, which than evolve into spiral waves.

The reasons why the presence of obstacles may lead to localized conduction block are twofold. First, narrow gaps present between nearby obstacles lead to a so-called source-sink mismatch: in the gap the wave conists of only a few excited points that serve as a current source, whereas for the wave to progress after the gap, a much larger 

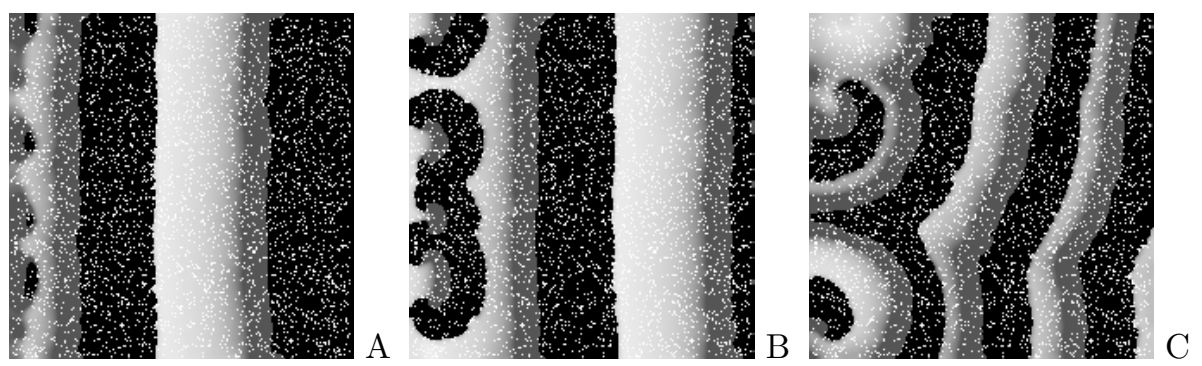

FIG. 3.3. Development of initial wave breaks (A), and subsequent spiral wave formation (B), and presence of stable spiral activity $(\mathrm{C})$ after applying three stimuli with a short stimulus period. Results are for a medium with $10 \%$ obstacles, $\mu_{1}=0.14$, and a stimulation period of 28 time units.

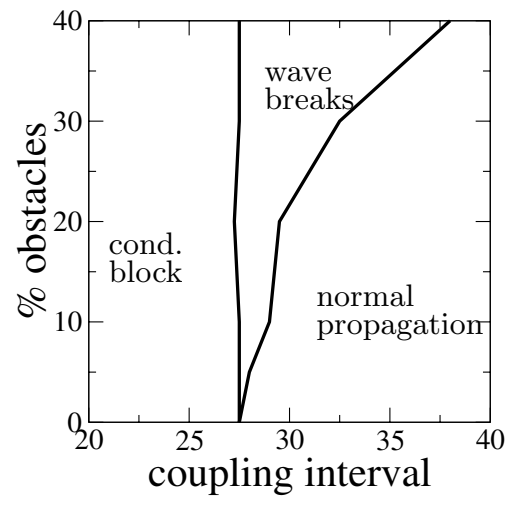

FIG. 3.4. Dependence of the vulnerable window in stimulation coupling interval space on the percentage of obstacles present. Results are for $\mu_{1}=0.14$.

number of points that serve as a current sink need to be excited. This may lead to local conduction block after the gap. In addition, clumps of small obstacles may form a larger obstacle interrupting the wave front as discussed in $[36,1,7,8]$. Figure 3.3 shows an example of the successful induction of spiral waves after application of three stimuli with a short coupling interval. In Figure 3.3(A) we can see that the third stimulus gives rise to a locally propagating and a locally blocked wave. In Figure 3.3(B) we can see how these broken wave fronts immediately curl around to form spiral waves. In Figure 3.3(C) the wave pattern is shown after 256 units of time (time is in dimensionless units) after the third stimulus. It can be seen that of the five spirals originally formed (Figure 3.3(B)), two are remaining; the others have disappeared through collisions with each other or the boundary of the medium. Note also that the spirals stay in the area of the medium where they originated.

In Figure 3.4 we show the dependence of the vulnerable window of coupling intervals that lead to wave breaks and spiral formation on the percentage of obstacles. We see that the lower border of the vulnerable window, below which the interval between stimuli is too short and wave propagation blocks, is independent of the number of obstacles present in the medium. It can also be seen that the upper border of the vulnerable window, above which normal wave propagation occurs, is strongly dependent on the percentage of obstacles. If the number of obstacles increases, the upper border of the vulnerable window goes up, increasing the width of the vulnerable window. 

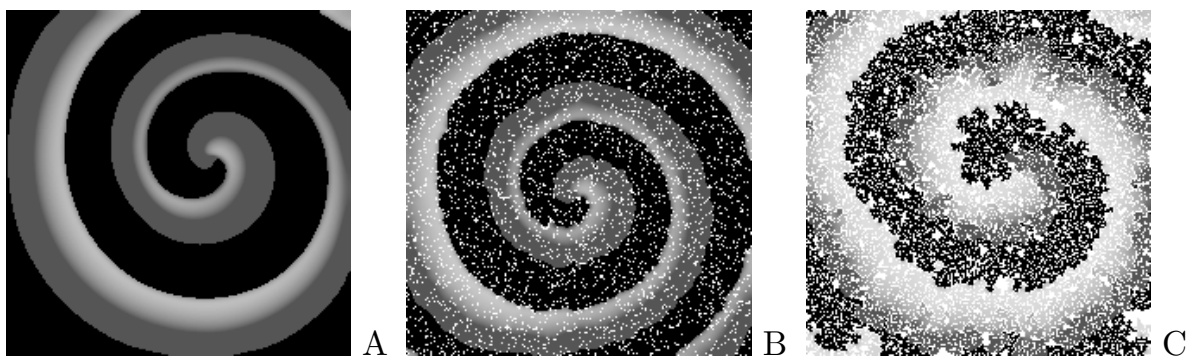

FiG. 3.5. Spiral wave rotation in a medium with $0 \%$ (A), $10 \%$ (B), or $30 \%$ (C) obstacles. Results are for $\mu_{1}=0.13$.

3.4. Effect of obstacles on spiral wave rotation. In this section we study the effect of obstacles on spiral wave rotation. Spirals are created by initializing the medium with a broken wave front that curls around to form a single spiral wave. Figure 3.5 shows stable spiral wave patterns for media with no obstacles (A), $10 \%$ obstacles (B), and 30\% obstacles (C). From Figure 3.5 it can be seen that increasing the percentage of obstacles leads to a less regularly shaped spiral wave with local disturbances in the wavefront; wavelength, however, does not seem to be affected by the percentage of obstacles.

To study in more detail the effect of randomly distributed obstacles on spiral wave dynamics, we computed how the main characteristics of spiral waves, namely $\mathrm{CV}$, action potential duration (duration of the excited state) (APD), diastolic interval (the interval between two successive action potentials) (DI), and period (APD+DI) of spiral wave rotation, depend on the percentage of obstacles. The results of these computations are shown in Figure 3.6. It can be seen that conduction velocity decreases approximately linearly as a function of the percentage of obstacles, whereas period, DI, and APD all increase in a similar nonlinear manner as a function of the percentage of obstacles. From these results it can also be understood why wavelength stays approximately the same for percentages of obstacles in the range from 0 to $30 \%$ : wavelength is the product of the period and the CV. For $0 \%$ obstacles, period is approximately 26.5 and $\mathrm{CV}$ is 1.24 , resulting in a wavelength of 32.9 ; for $30 \%$ obstacles, period is 38.6 and CV 0.87 , resulting in a wavelength of 33.6 , which is approximately the same as for $0 \%$ obstacles. In other words, the decrease in CV is compensated by an equally sized increase in period, leading to similar wavelengths. For 35\% obstacles and more, this effect no longer holds: CV keeps decreasing linearly with the percentage of obstacles, whereas period increases more than linearly with the percentage of obstacles, causing wavelength to increase: for $35 \%$ obstacles, period is $64.2, \mathrm{CV}$ is 0.82 , and therefore wavelength is 52.6 , which is significantly more than for 0 and $30 \%$ of obstacles.

Note that Figure 3.6 shows average values of spiral wave characteristics. Individual values of spiral wave characteristics vary, depending on the particular pattern in which the obstacles are distributed over the excitable medium. This variation of spiral wave characteristics as a function of obstacle pattern can have important consequences for spiral wave dynamics, and will be further illustrated in section 4.2.

3.5. Effect of obstacles on spiral breakup in two dimensions. Now we investigate the influence of randomly distributed small obstacles on spiral wave breakup in 2D media. Spiral wave breakup is the process of fragmentation of a single spiral wave into a spatio-temporally chaotic wave pattern [35, 9, 20,23,13], which is thought to underlie another type of dangerous cardiac arrhythmia called fibrillation. To study 

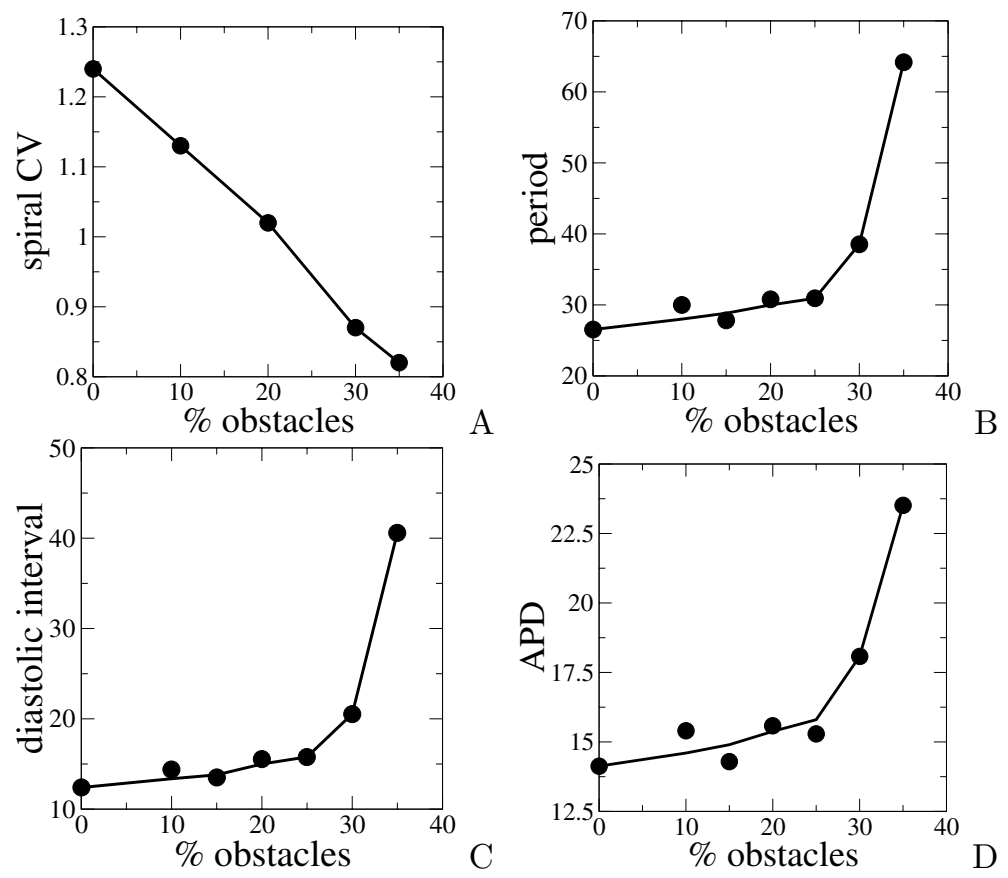

FIG. 3.6. Dependence of spiral conduction velocity (A), period (B), diastolic interval (C), and action potential duration $(A P D,(D))$ on the percentage of obstacles present. Results are for $\mu_{1}=0.13$.

the effect of obstacles on the occurrence of spiral breakup, we used parameter values for which spiral breakup is observed in a homogeneous medium without obstacles, and studied how the presence of obstacles affect the breakup phenomenon. We started our simulations with a broken wave front that curls around to form a single spiral wave. In Figure 3.7 we see the time evolution of spiral wave patterns in $2 \mathrm{D}$ media with $0 \%$ (top row), $10 \%$ (middle row), and $30 \%$ (bottom row) obstacles for a parameter value of $\mu_{1}=0.09$. In all three cases, at time $T=160$ (first column) an intact spiral wave is present. In the top row, the first wave break occurs at time $T=428$ (second column), and after that we can see a turbulent wave pattern develop (third and fourth columns). In the middle row at time $T=428$ (second column) the spiral wave is still intact, but at time $T=832$ (third column) the first wave break occurs, and this wave break also develops into a chaotic wave pattern (fourth column). In the bottom row no wave breaks occur, and the spiral wave is still stable at time $T=1600$ (fourth column). These results suggest that increasing the percentage of obstacles suppresses spiral wave breakup: $10 \%$ of obstacles postponed the occurrence of the first wave break, and $30 \%$ of obstacles completely suppressed spiral breakup.

In the Aliev-Panfilov model the occurrence of spiral breakup is linked to the value of the parameter $\mu_{1}$. If $\mu_{1}$ is below some critical value, spiral breakup occurs; above the critical value spiral waves are stable. Therefore, we further investigate whether increasing the percentage of obstacles indeed suppresses spiral wave breakup, by performing a series of simulations for different values of $\mu_{1}$ in combination with different percentages of obstacles. Figure 3.8 displays the dependence of the critical value of $\mu_{1}$ on the percentage of obstacles. We can indeed see that increasing the percentage of obstacles decreases the window in parameter space for which spiral breakup occurs.

Now we would like to find out why spiral breakup disappears if the percentage of 

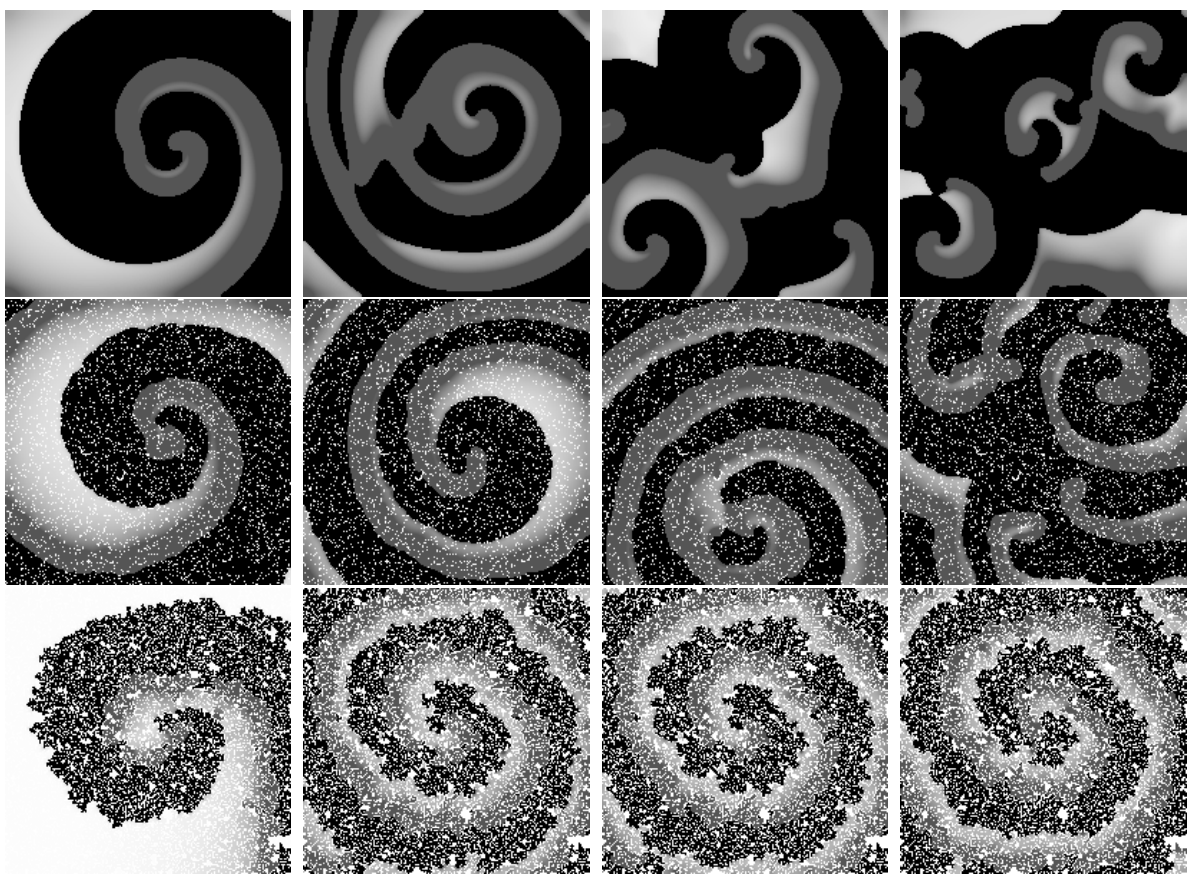

FIG. 3.7. Time evolution of $2 D$ spiral wave patterns. Top row: medium with no obstacles present. Middle row: medium with $10 \%$ obstacles. Bottom row: medium with $30 \%$ obstacles. First column: wave patterns at time $T=160$. Second column: wave patterns at time $T=428$. Third column: wave patterns at time $T=832$. Fourth column: wave patterns at time $T=1600$. Results are for $\mu_{1}=0.09$

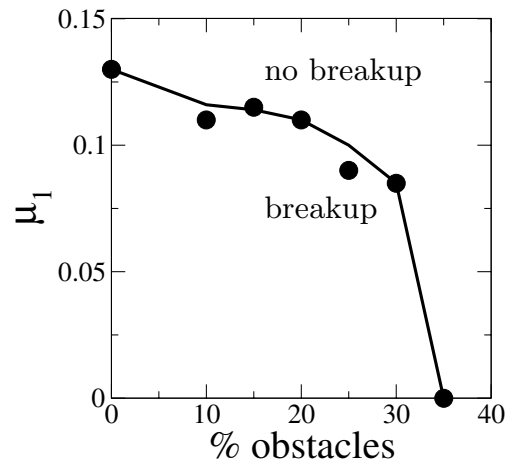

FIG. 3.8. Dependence of the critical value of $\mu_{1}$ on the percentage of obstacles present in $2 D$ media.

obstacles increases. The mechanism of spiral breakup in this model is the presence of a steep restitution curve [34]. The restitution curve describes the relationship between APD and the duration of the preceding DI. Typically, APD decreases if the DI is shorter and cells have had less time to recover. It can be mathematically proven that if the restitution relation has a slope steeper than 1, a flip bifurcation or period doubling bifurcation occurs, leading to alternations of long and short action potentials and long and short DIs. In Figure 3.9 a typical restitution curve is shown for $\mu_{1}=0.09$; for comparison, a line with a slope 1 is added. It can be seen that the restitution slope 


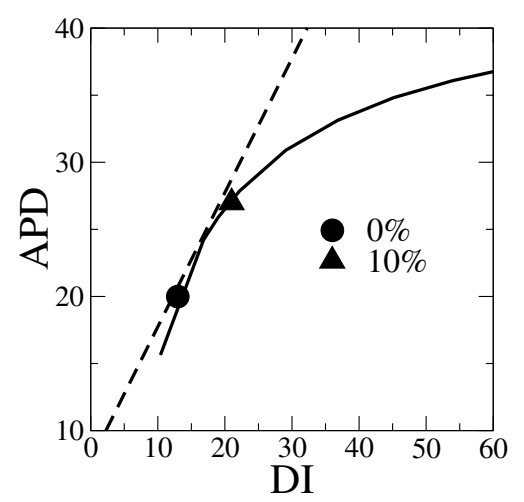

FIG. 3.9. Restitution curve of the relationship between action potential duration (APD) and preceding diastolic interval (DI) for $\mu_{1}=0.09$. For comparison, a line with a slope of 1 is drawn. Two points are added corresponding to spiral waves rotating in $2 D$ media with 0 or $30 \%$ obstacles, respectively.
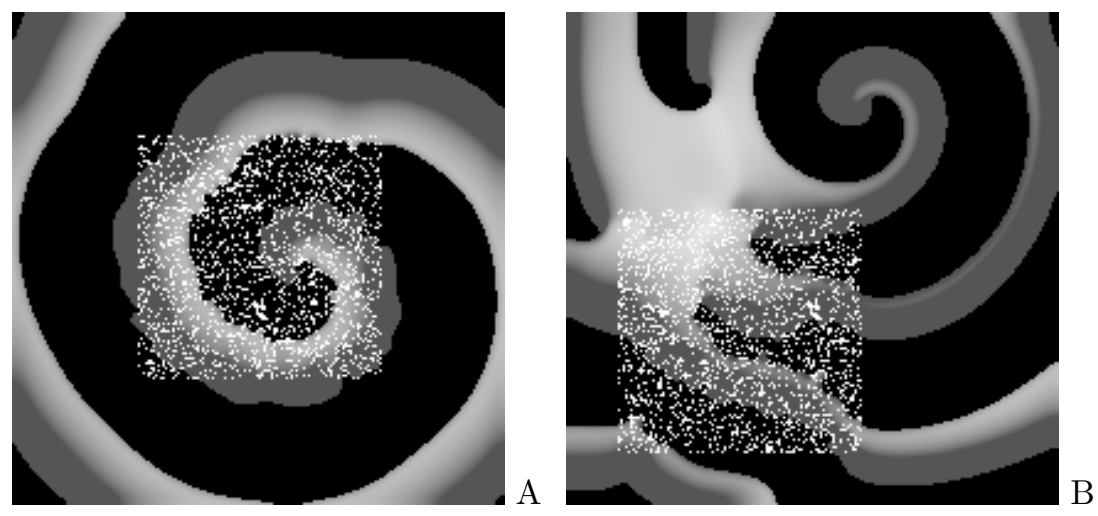

FIG. 3.10. $2 D$ media in which $30 \%$ obstacles are present only in a localized patch. (A) Spiral tip rotates inside the area with obstacles and is stable. (B) Spiral tip rotates outside the area with obstacles and undergoes breakup. In both cases $\mu_{1}=0.11$.

is steepest for short DIs and hence high excitation frequencies. Spiral waves typically rotate with a high frequency, and if for this excitation frequency the restitution slope exceeds 1, a flip bifurcation occurs, APDs alternate, and if locally a very short action potential arises, localized conduction block and spiral wave breakup can occur.

In the previous section on the influence of obstacles on spiral wave rotation, we showed that increasing the percentage of obstacles leads to an increase of spiral wave period, DI, and APD. In Figure 3.9 we show how these effects lead to the suppression of spiral wave breakup. On the restitution curve two points are drawn. The circle is for a spiral rotating in a medium with no obstacles; the spiral has a short DI and lies on the part of the restitution curve that has a slope steeper than 1: spiral breakup will occur. The triangle is for a spiral rotating in a medium with $30 \%$ obstacles; the spiral therefore has a longer DI and lies on the part of the restitution curve that has a slope less than 1: the spiral wave will stay stable. From this we can derive the following mechanism for spiral wave breakup suppression by obstacles: increasing the percentage of obstacles leads to an increase in period, APD, and DI that causes a shift along the restitution curve, away from the steep part where breakup occurs.

In Figure 3.10 we show 2D media for which obstacles are present in only part 
of the medium. Parameters are the same in the two figures: $\mu_{1}=0.11$ and locally $30 \%$ obstacles, the only difference being the location of the patch with obstacles relative to the tip of the spiral. In Figure 3.10(A) the spiral tip, which is the organizing center of a spiral wave and determines the rotation speed of the spiral, resides in the area with obstacles; as a consequence, the obstacles slow down rotation and the spiral stays stable. In Figure 3.10(B), the spiral tip is outside the area with obstacles; therefore the obstacles cannot slow down spiral wave dynamics, and spiral breakup occurs. These results indicate that for obstacles to stabilize spiral wave dynamics they do not need to be present in the whole medium; instead, it is important that the tip of the spiral be inside the region where the obstacles are present.

4. Wave propagation in 3D media. Next we would like to investigate the effect of obstacles on 3D wave propagation. However, since there are many similarities between $2 \mathrm{D}$ and $3 \mathrm{D}$ wave propagation, we will not study all aspects that were studied in $2 \mathrm{D}$ but rather study the influence of obstacles on vulnerability and scroll wave (3D spiral wave) breakup in 3D. We find both differences and similarities in $2 \mathrm{D}$ and $3 \mathrm{D}$ media, and we explain the mechanism behind the differences.

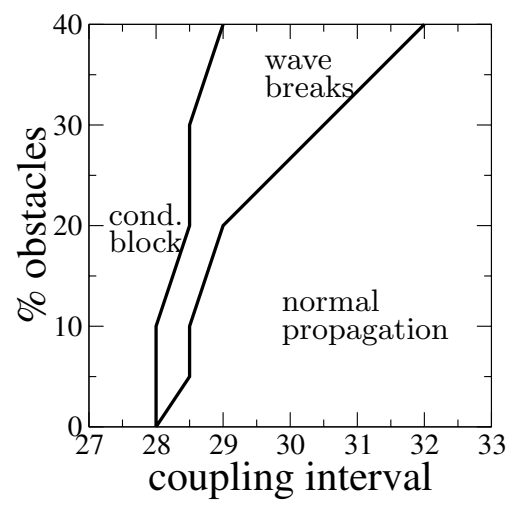

FIG. 4.1. Dependence of the vulnerable window in stimulation coupling interval space on the percentage of obstacles present in $3 D$ media. Results are for $\mu_{1}=0.14$ and a coupling strength in the $z$ direction that is $1 / 9$ of that in the $x$ and $y$ directions.

4.1. Vulnerability in the presence of obstacles in three dimensions. In this section we study whether the presence of obstacles also leads to an increased vulnerability to spiral wave formation in $3 \mathrm{D}$ excitable media. Two series of simulations were performed. In the first series, coupling between excitable cells was equal in the $x$, $y$, and $z$ directions. In the second series, coupling strength in the $z$ direction is $1 / 9$ of that in the $x$ and $y$ directions, reflecting the layer structure of cardiac tissue [29] but ignoring many other details of cardiac tissue structure in order to keep the problem close to that considered in two dimensions. In the first series of experiments we did not observe the formation of wave breaks or spiral waves. In the second series of experiments (with less strong coupling in the $z$ direction) spiral waves could be induced. The dependence of the vulnerable window on the percentage of obstacles for this particular case is shown in Figure 4.1. From a comparison of Figures 4.1 and 3.4 it can be seen that the vulnerable window for scroll wave formation is much smaller than in 2D media and is shifted towards higher percentages of obstacles.

Both the fact that scroll waves could not be induced in the first series of experiments and that in the second series the vulnerable window was much narrower than in 
two dimensions can be explained as follows: in two dimensions, when a wave is locally blocked by a clump of obstacles or a small gap, a wave break always occurs. However, in three dimensions, the random pattern of obstacles is different in the different layers of the medium. Therefore, often when conduction is blocked in one layer of the medium it can still propagate in the adjacent layers. As a consequence, wave front disturbances get quickly restored from other layers, due to the electrotonic interaction between layers. We found that for conduction block to arise in three dimensions the coupling between layers should be less strong than within layers in order for wave front defects not to be restored immediately from other layers. In addition, we found that higher percentages of obstacles are required than in two dimensions to get the same amount of vulnerability, also indicating that sustained wave breaks are harder to create in three dimensions. Higher percentages of obstacles result in larger clumps, leading to larger wave breaks that can less easily be restored; lead to larger numbers of gaps and wave breaks, which might not all be restored; and increase the likelihood of conduction block co-occurring in neighboring layers, again making wavefront deformations less easy to restore.

It should be noted that the combined effect of anisotropy and obstacles on wave propagation is a complex issue that requires further investigation; however, this is not the goal of the present study.
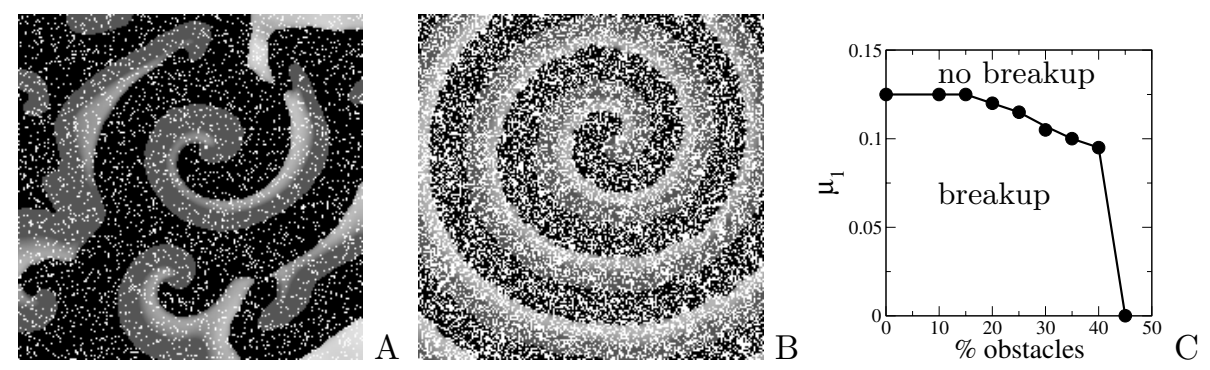

FIG. 4.2. A Wave patterns at time $\mathrm{T}=2600$ for $\mu_{1}=0.11$ and $10 \%$ or $\mathrm{B} 40 \%$ of obstacles. The shown wave patterns are from a $2 D$ cross-section at the middle layer of the $3 D$ medium. $\mathrm{C}$ Dependence of the critical value of $\mu_{1}$ below which breakup occurs on the percentage of obstacles in $3 D$.

4.2. Effect of obstacles on spiral breakup in three dimensions. Finally, we investigate whether randomly distributed small obstacles also suppress spiral wave breakup in 3D media. In Figure 4.2(A) and (B), 2D cross sections of 3D wave patterns are shown. In Figure 4.2(A) we see a turbulent wave pattern that arises after spiral breakup in a 3D medium with $10 \%$ obstacles. In Figure $4.2(\mathrm{~B})$ we see a stable spiral wave in a 3D medium for the same parameter settings as in Figure 4.2(A) but with $40 \%$ obstacles. Figure $4.2(\mathrm{C})$ displays the dependence of the critical value of $\mu_{1}$ below which spiral breakup occurs on the percentage of obstacles. We can see that increasing the percentage of obstacles decreases the window in parameter space for which spiral breakup occurs, similarly to what was observed for 2D media. However, from a comparison of Figures 4.2(C) and 3.8, it can be seen that in three dimensions a larger percentage of obstacles is required to obtain the same decrease in the critical value of $\mu_{1}$ : the $2 \mathrm{D}$ curve for the critical value of $\mu_{1}$ decreases faster as a function of the percentage of obstacles than the 3D curve. In addition, in three dimensions the critical value for $\mu_{1}$ goes to zero for $45 \%$ obstacles, whereas in two dimensions the critical value for $\mu_{1}$ already goes to zero for $35 \%$ obstacles. 


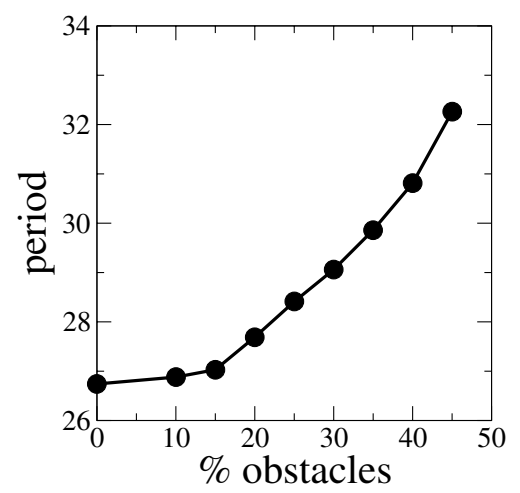

Fig. 4.3. Scroll wave period as a function of the percentage of obstacles in three dimensions. Results are for $\mu_{1}=0.13$.

To investigate why the effect of obstacles on spiral breakup is less pronounced in three than in two dimensions, we measured the dependence of scroll wave period, DI, and APD on the percentage of obstacles in 3D excitable media. Figure 4.3 shows the dependence of period on the percentage of obstacles in three dimensions. It can be seen that the period increases in a nonlinear fashion as a function of the percentage of obstacles, similar to the case in two dimensions. However, from a comparison of Figures 4.3 and 3.6(B), it can be seen that the increase in period is much less pronounced in 3D than in 2D excitable media: for 35\% obstacles in two dimensions, the period has increased from approximately 26.5 (for $0 \%$ obstacles) to 64.2 , whereas in three dimensions, the period has increased from the same 26.5 to only 29.9. Similar results were obtained for DI and APD (data not shown). Thus, to get the same increase in DI and shift a spiral wave to the part of the restitution curve with a slope less than 1, a larger percentage of obstacles is needed. The next question now is why a certain percentage of obstacles leads to much less increase in period, DI, and APD in $3 \mathrm{D}$ than in $2 \mathrm{D}$ media.

To explain this difference, we repeated the 3D simulation for a value of $\mu_{1}=0.13$ and $30 \%$ obstacles, but now we decoupled the layers of the 3D slab in the $z$ direction. This results in 76 independent $2 \mathrm{D}$ simulations that have an obstacle pattern corresponding to those of the different layers in the 3D medium that we simulated previously. We determined the average period of spiral wave rotation in each of the 76 layers and found that there was a large variability in spiral wave period between layers, with a lowest period of 29.47 (layer 32), a highest period of 78.05 (layer 63), a mean period of 41.85 , and a standard deviation of 9.98. These results suggest that due to different spatial distribution of obstacles in the different layers, the spiral waves have substantially different periods. As was shown in [33,37], if different layers with different periods of spiral rotation are coupled into a heterogeneous 3D medium, the medium will be driven by the layer with the fastest spiral, due to the electrotonic interactions between the different layers.

In order to check whether this effect also holds for media with inexcitable obstacles, we simulated spiral wave rotation for $\mu_{1}=0.13$ in a $100 \times 100 \times 60$ medium consisting of three different but fully coupled regions each of 20 layers in height, with the outer two regions containing no obstacles and the inner region containing $0 \%$, $10 \%, 20 \%, 30 \%$, or $40 \%$ obstacles. In Figure 4.4 it can be seen that the period of spiral wave rotation in these media is independent of the percentage of obstacles in 


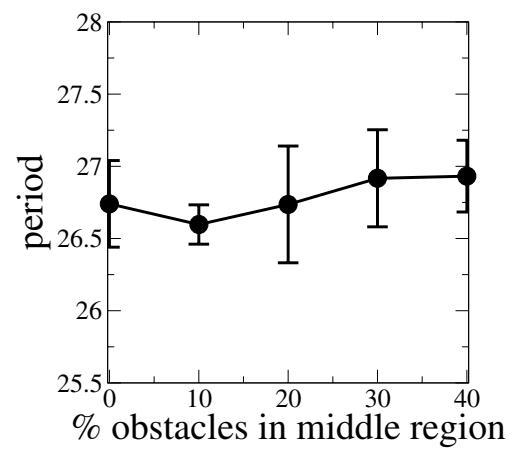

FIG. 4.4. Period of scroll wave rotation as a function of the percentage of obstacles in the middle region of a $3 D$ excitable medium that has no obstacles in the outer two regions of the medium. To illustrate that periods do not differ significantly for different percentages of obstacles, mean values and standard deviations are shown. Results are for $\mu_{1}=0.13$.
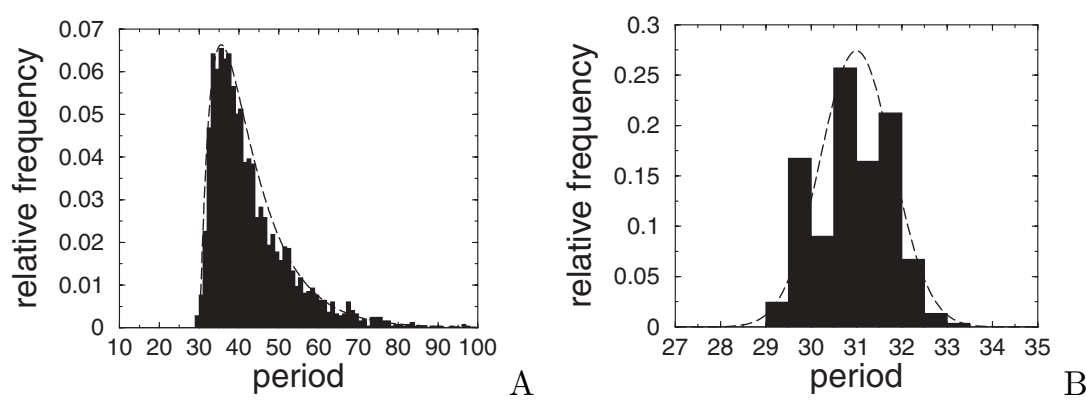

FIG. 4.5. (A) Relative frequency distribution of the spiral wave period for 2477 simulations. (B) Relative frequency distribution of the minimum period out of 76 periods for 2000 experiments. For clarity fitted idealized frequency distributions are also shown. Results are for $\mu_{1}=0.13$ and $30 \%$ obstacles.

the middle region of the medium and equal to the spiral wave period in a medium without obstacles $(0 \%$ obstacles in middle region), confirming that the effect (of the pace being set by the layer with the fastest spiral) also holds in the case of obstacles.

Going back to our experiment with the uncoupled layers, we can indeed see that in the completely coupled 3D simulation the scroll wave period is 29.06 , which is very close to the lowest spiral wave period recorded in our uncoupled 3D simulation.

The fact that the 3D spiral period approximately equals the minimum period of the 2D layers constituting the 3D medium allows for the following statistical interpretation [43]: if $f(x)$ is the density distribution function of $2 \mathrm{D}$ spiral wave periods, and $F(x)=\int_{-\infty}^{x} f(u) d u$ is the distribution function for the probability that a period is less than $x$, then the probability that at least one in $N$ randomly selected patterns has a period less than $x$ is $\Phi(x)=1-(1-F(x))^{N}$. Therefore, the density distribution function for the minimum period out of $N$ periods and hence for the expected period of a 3D scroll wave in a medium with $N$ layers is $\phi(x)=\frac{d \Phi(x)}{d x}=N(1-F(x))^{N-1} f(x)$. Since it is not possible to derive the density distribution function $f(x)$ analytically, we estimated the density distributions $f(x)$ and $\phi(x)$ by Monte Carlo simulation. Figure 4.5(A) shows the relative frequency distribution (estimate of $f(x)$ ) of spiral wave periods found in 2477 simulations for $\mu_{1}=0.13$ and $30 \%$ obstacles in different patterns. The distribution has a mean of 42.77 and a variance of 9.96. To mimic 
our 3D simulation consisting of 76 layers, with the fastest layer determining scroll wave period, we randomly drew 76 simulations from the total of 2477 , determined the minimum period, and repeated this experiment 2000 times. From this we obtained a relative frequency distribution for the minimum (out of 76) period (estimate of $\phi(x)$ ) (Figure 4.5(B)). This distribution has a mean of 30.93 and a variance of 0.81 . From a comparison of Figures 4.5(A) and (B) we can see that the expected value for the period of a $3 \mathrm{D}$ scroll wave (the mean of the minimum period distribution) is almost $30 \%$ shorter than that of a $2 \mathrm{D}$ spiral wave (the mean of the normal period distribution). We can also see that the variance of the $3 \mathrm{D}$ period is much smaller than that of the 2D period. Note that we found a period of 38.48 for the 2D spiral wave and a period of 29.06 for the 3D scroll wave, close to the mean values of the normal and minimum period distributions, respectively (Figure 4.5(A) and (B)).

Summarizing, the shorter spiral wave period in $3 \mathrm{D}$ than in $2 \mathrm{D}$ media in the presence of obstacles is caused by the fact that in three dimensions, the scroll wave period is determined by the minimum period of multiple 2D layers, which, as follows from our statistical analysis, will on average be smaller than a single, normal 2D period.

5. Discussion. We studied the effects of randomly distributed small nonexcitable obstacles on wave propagation in excitable media. In 2D media, we showed that increasing the percentage of obstacles leads to a linear decrease in conduction velocity, which can be explained simply by the smaller number of excitable cells contributing to the propagating wave front. We also investigated the effect of obstacles on the eikonal curvature relation for excitable media. We found that the linear dependence of wave speed on curvature also holds in the presence of obstacles, but that the effective diffusion coefficient decreases with an increase in the percentage of obstacles.

We showed that the vulnerability of the medium for wave breaks and spiral wave formation increases as a function of the percentage of obstacles. This agrees well with clinical data on the increased occurrence of cardiac arrhythmias in the presence of increased amounts of fibrotic tissue in the heart $[11,18]$. In addition, we showed that an increase in the percentage of obstacles leads to a suppression of spiral wave breakup. This result, which at first may seem counterintuitive, we explained by showing that an increased number of obstacles slows down spiral wave dynamics, leading to longer period, action potential duration, and diastolic interval, thus suppressing the steep restitution breakup mechanism present in this model.

The latter result might agree less well with clinical data, as increased amounts of fibrosis are correlated both with arrhythmias caused by the rotation of a single spiral wave (tachycardia) and with arrhythmias generated by the chaotic excitation patterns that arise after spiral wave fragmentation (fibrillation) [45, 17]. In this context it is important to realize that obstacles suppress spiral breakup caused by the steep restitution mechanism, but that this need not be the case for other possible mechanisms of spiral wave fragmentation $[31,4,40]$. Indeed, the variation of spiral wave periods induced by different percentages and patterns of obstacles through the thickness of a 3 D excitable medium may potentially result in alternative mechanisms of scroll wave breakup involving filament twist induced by the transversal heterogeneity, as shown in [37]. However, we did not observe such dynamics in our current study, probably because either spiral wave period differences were too small or particular periods extended over only one or a few layers, thus not inducing substantial filament twist. In addition, it is important to note that the disease states that lead to extensive fibrosis in the heart often also lead to changes in both the excitable properties of cardiac 
cells and their coupling to other cells [42, 21], which are not captured in our simple model of excitable media but that may very well lead to breakup mechanisms unique to cardiac excitable tissue.

We extended our study to 3D excitable media. We showed that obstacles also lead to vulnerability for wave breaks and scroll wave formation in three dimensions, but that this effect is less strong than in two dimensions. For wave breaks and spiral waves to be induced, coupling in the $z$ direction had to be less strong than in the $x$ and $y$ directions, and higher percentages of obstacles were required for the same amount of vulnerability as in two dimensions. This difference can be explained by the fact that in three dimensions the wave front can also progress in the direction of the third dimension, causing wave breaks in one layer to be repaired by normally progressing wave fronts in neighboring layers. Based on these results, we can hypothesize that the effect of fibrose tissue on the initiation of cardiac arrhythmias should be more pronounced in the atria (thin, semi-2D) than in the ventricles (thick, 3D) of the heart. Note, however, that these results should be verified in more detailed electrophysiologically and anatomically adequate models of cardiac tissue before they can be reliably extended to a clinical context.

Finally, we demonstrated that the stabilizing effect of obstacles on spiral wave dynamics also occurs in 3D media, but again this effect is less pronounced than in two dimensions. We demonstrated that this difference is caused by the fact that in three dimensions, the scroll wave period is determined by the minimum period of multiple 2D spirals in the different layers of the $3 \mathrm{D}$ medium. This result may indicate that in the presence of fibrotic tissue the period of arrhythmias might be shorter in thin sections of the heart than in thick sections.

Note that the effects we studied here are relevant not only for cardiac tissue but also for wave propagation in other excitable media. As a few examples, research has been performed on the influence of patterned heterogeneities and obstacles on wave propagation both in the Belousov-Zhabotinsky reaction [30, 41] and in CO oxidation on Pt surfaces [5], including the influence of heterogeneities on spiral wave formation and stability.

Acknowledgments. We are grateful to Dr. J. de Bakker and Dr. E. Belitser for valuable discussions and help in this research.

\section{REFERENCES}

[1] K. I. Agladze, J. P. Keener, S. C. Müller, and A. V Panfilov, Rotating spiral waves created by geometry, Science, 264 (1994), pp. 1746-1748.

[2] R. R. Aliev and A. V. Panfilov, A simple two-variable model of cardiac excitation, Chaos Solitons Fractals, 7 (1996), pp. 293-301.

[3] M. A. Allessie, F. I. M. Bonke, and F. J. G. Schopman, Circus movement in rabbit atrial muscle as a mechanism of tachycardia, Circ. Res., 33 (1973), pp. 54-62.

[4] M. BAËR AND M. OR-GUIL, Alternative scenarios of spiral breakup in a reaction-diffusion model with excitable and oscillatory dynamics, Phys. Rev. Lett., 82 (1999), pp. 1160-1163.

[5] M. Bär, A. K. Bangia, I. G. Kevrekidis, G. Haas, H. H. Rotermund, and G. Ertl, Composite catalyst surfaces: Effect of inert and active heterogeneities on pattern formation, J. Phys. Chem., 100 (1996), pp. 19106-19117.

[6] M. Bär, E. Meron, AND C. UtZny, Pattern formation on anisotropic and heterogeneous catalytic surfaces, Chaos, 12 (2002), pp. 204-214.

[7] C. Cabo, A. M. Pertsov, W. T. Baxter, J. M. Davidenko, R. A. Gray, and J. Jalife, Wave-front curvature as a cause of slow conduction and block in isolated cardiac muscle, Circ. Res., 75 (1994), pp. 1014-1028. 
[8] C. Cabo, A. M. Pertsov, J. M. Davidenko, W. T. Baxter, R. A. Gray, and J. Jalife, Vortex shedding as a precursor of turbulent electrical activity in cardiac muscle, Biophys. J., 70 (1996), pp. 1105-1111.

[9] M. Courtemanche And A. T. Winfree, Re-entrant rotating waves in a Beeler-Reuter based model of two-dimensional cardiac activity, Internat. J. Bifur. Chaos Appl. Sci. Engrg., 1 (1991), pp. 431-444.

[10] J. M. Davidenko, A. M. Pertsov, R. Salomonsz, W. Baxter, and J. Jalife, Stationary and drifting spiral waves of excitation in isolated cardiac muscle, Nature, 355 (1991), pp. 349-351.

[11] J. M. T. De Bakker, F. J. L. Van Capelle, and M. J. Janse, Reentry as a cause of ventricular tachycardia in patients with chronic ischemic heart disease: Electrophysiologic and anatomic correlation, Circulation, 68 (1988), pp. 518-533.

[12] V. G. FASt And A. G. KLÉBER, Role of wavefront curvature in propagation of cardiac impulse, Cardiovasc. Res., 33 (1997), pp. 258-271.

[13] A. Garfinkel, Y. H. Kim, O. Voroshilovsky, Z. Qu, J. R. Kil, M. H. Lee, H. S. KaraGueuzian, J. N. Weiss, And P. S. Chen, Preventing ventricular fibrillation by flattening cardiac restitution, Proc. Natl. Acad. Sci. USA, 97 (2000), pp. 6061-6066.

[14] G. Gerisch, Standienspezifische aggregationsmuster bei Dictyostelium discoideum, Wilhelm Roux Arch. Entwick Org, 156 (1965), pp. 127-144.

[15] N. A. Gorelova And J. J. Bures, Spiral waves of spreading depression in the isolated chicken retina, J. Neurobiol., 14 (1983), pp. 353-363.

[16] P. Grindrod, Patterns and Waves: The Theory and Application of Reaction-Diffusion Equations, Claredon Press, Oxford, 1991.

[17] H. Hayashi, C. Wang, Y. Miyauchi, C. Omichi, H. N. Pak, S. Zhou, T. Ohara, W. J. Mandel, S. F. Lin, M. C. Fishbein, P. C. Chen, and H. S. Karagueuzian, Aging-related increase to inducible atrial fibrillation in the rat model, J. Cardiovasc. Electrophysiol., 13 (2002), pp. 801-808.

[18] H. H. Hsia and F. E. Marchlinski, Characterization of the electroanatomic substrate for monomorphic ventricular tachycardia in patients with nonischemic cardiomyopathy, Pacing Clin. Electrophysiol., 25 (2002), pp. 1114-1127.

[19] R. Imbinl and G. ERTL, Oscillatory kinetics in heterogeneous catalysis, Chem. Rev., 95 (1995), pp. $697-733$.

[20] H. Ito and L. Glass, Spiral breakup in a new model of discrete excitable media, Phys. Rev. Lett., 66 (1991), pp. 671-674.

[21] H. J. Jongsma And R. Wilders, Gap junctions in cardiovascular disease, Circ. Res., 86 (2000), pp. 1193-1197.

[22] B. I. Jugdutt, Remodelling of the myocardium and potential targets in the collagen synthesis pathways, Curr. Drug Targets Cardiovasc. Haematol. Disord., 3 (2003), pp. 1-30.

[23] A. KARMA, Spiral breakup in model equations of action potential propagation in cardiac tissue, Phys. Rev. Lett., 71 (1993), pp. 1103-1106.

[24] J. P. KeEner, An eikonal equation for action potential propagation in myocardium, J. Math. Biol., 29 (1991), pp. 629-651.

[25] J. P. KeEner, Propagation of waves in an excitable medium with discrete release sites, SIAM J. Appl. Math., 61 (2000), pp. 317-334.

[26] V. I. KRINSKY, Mathematical models of cardiac arrhythmias (spiral waves), Pharm. Ther. B, 3 (1978), pp. 539-555.

[27] V. I. KRINSKY, Autowaves: Results, problems, outlooks, in Self-Organization. Autowaves and Structures Far from Equilibrium, V. I. Krinsky, ed., Springer-Verlag, Heidelberg, 1984, pp. 9-18.

[28] J. Lechleiter, S. Girard, E. Peralta, and D. Clapham, Spiral calcium wave propagation and annihilation in Xenopus laevis oocytes, Science, 252 (1991), pp. 123-126.

[29] I. J. LeGrice, P. J. Hunter, And B. H. Smail, Laminar structure of the heart. II. Mathematical model, Am. J. Physiol., 269 (1995), pp. H571-H582.

[30] N. Manz, V. A. Davydov, V. S. Zykov, And S. C. Müller, Excitation fronts in a spatially modulated light-sensitive Belousov-Zhabotinsky system, Phys. Rev. E, 66 (2002), 036207$1-5$.

[31] A. F. M. Marée And A. V. Panfilov, Spiral breakup in excitable tissue due to lateral instability, Phys. Rev. Lett., 78 (1997), pp. 1819-1822.

[32] E. Meron, Pattern formation in excitable media, Phys. Rep., 218 (1992), pp. 1-66.

[33] A. V. Panfilov, Three-dimensional vortices in active media, in Nonlinear Wave Processes in Excitable Media, A. V. Holden, M. Markus, and H. G. Othmer, eds., Plenum Press, New York, 1991, pp. 361-382. 
[34] A. V. PAnfilov, Spiral breakup in an array of coupled cells: The role of the intercellular conductance, Phys. Rev. Lett., 88 (2002), paper 118101.

[35] A. V. Panfilov And A. V. Holden, Self-generation of turbulent vortices in a two-dimensional model of cardiac tissue, Phys. Lett. A, 147 (1990), pp. 463-466.

[36] A. V. Panfilov and J. P. Keener, Effects of high frequency stimulation on cardiac tissue with an inexcitable obstacle, J. Theoret. Biol., 163 (1993), pp. 439-448.

[37] A. V. Panfilov and J. P. Keener, Twisted scroll waves in heterogeneous excitable media, Internat. J. Bifur. Chaos Appl. Sci. Engrg., 3 (1993), pp. 445-450.

[38] A. Pertsov, Scale of geometric structures responsible for discontinuous propagation in myocardial tissue, in Discontinuous Conduction in the Heart, P. M. Spooner, R. W. Joyner, and J. Jalife, eds., Futura Publishing Company, Armonk, NY, 1997, pp. 273-293.

[39] A. M. Pertsov, J. M. Davidenko, R. SAlomontsz, W. Baxter, and J. Jalife, Spiral waves of excitation underlie reentrant activity in isolated cardiac muscle, Circ. Res., 72 (1993), pp. 631-650.

[40] B. Sandstede And A. Scheel, Absolute versus convective instability of spiral waves, Phys. Rev. E, 62 (2000), pp. 7708-7714.

[41] O. Steinbock, P. Kettunen, and K. Showalter, Anisotropy and spiral organizing centers in patterned excitable media, Science, 269 (1995), pp. 1857-1860.

[42] B. Swyngedauw, Molecular mechanisms of myocardial remodeling, Physiol. Rev., 79 (1999), pp. $215-262$.

[43] K. H. W. J. Ten Tusscher and A. V. Panfilov, Influence of nonexcitable cells on spiral breakup in two-dimensional and three-dimensional excitable media, Phys. Rev. E, 68 (2003), paper 062902.

[44] A. M. Varnava, P. M. Elliott, N. Mahon, M. J. Davies, and W. J. McKenna, Relation between myocyte disarray and outcome in hypertrophic cardiomyopathy, Am. J. Cardiol., 88 (2001), pp. 275-279.

[45] T. J. WU, J. J. C. ONG, AND C. HwAng, The characteristics of wave fronts during ventricular fibrillation in human hearts with dilated cardiomyopathy: Role of increased fibrosis in the generation of reentry, J. Am. Coll. Cardiol., 32 (1998), pp. 187-196.

[46] A. N. Zaikin And A. M. Zhabotinsky, Concentration wave propagation in two-dimensional liquid-phase self-organising system, Nature, 225 (1970), pp. 535-537.

[47] V. S. ZyKov, Analytical evaluation of the dependence of the speed of an excitation wave in a two dimensional excitable medium on the curvature of its front, Biophys., 25 (1980), pp. 906-911.

[48] V. S. Zykov, Simulation of Wave Processes in Excitable Media, Manchester University Press, Manchester, UK, 1987. 